\title{
Altered glycosylation of complexed native IgG molecules is associated with disease activity of systemic lupus erythematosus
}

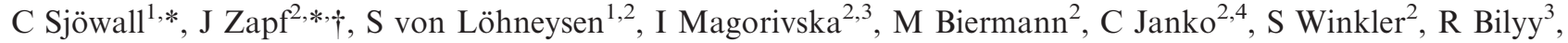 \\ G Schett ${ }^{2}$, M Herrmann ${ }^{2, *}$ and LE Muñoz ${ }^{2, *}$ \\ ${ }^{1}$ Rheumatology/AIR, Department of Clinical and Experimental Medicine, Linköping University, Linköping, Sweden; ${ }^{2}$ Department for Internal \\ Medicine 3 and Institute for Clinical Immunology, Friedrich-Alexander University of Erlangen-Nuremberg (FAU), Germany; ${ }^{3}$ Institute of Cell \\ Biology, National Academy of Sciences of Ukraine, Lviv, Ukraine; and ${ }^{4}$ Department of Otorhinolaryngology, Head and Neck Surgery, Section for \\ Experimental Oncology and Nanomedicine (SEON), University Hospital Erlangen, Germany
}

In addition to the redundancy of the receptors for the Fc portion of immunoglobulins, glycans result in potential ligands for a plethora of lectin receptors found in immune effector cells. Here we analysed the exposure of glycans containing fucosyl residues and the fucosylated tri-mannose N-type core by complexed native IgG in longitudinal serum samples of wellcharacterized patients with systemic lupus erythematosus. Consecutive serum samples of a cohort of 15 patients with systemic lupus erythematosus during periods of increased disease activity and remission were analysed. All patients fulfilled the 1982 American College of Rheumatology classification criteria. Sera of 15 sex- and age-matched normal healthy blood donors served as controls. The levels and type of glycosylation of complexed random $\mathrm{IgG}$ was measured with lectin enzyme-immunosorbent assays. After specifically gathering IgG complexes from sera, biotinylated lectins Aleuria aurantia lectin and Lens culinaris agglutinin were employed to detect IgG-associated fucosyl residues and the fucosylated tri-mannose $\mathrm{N}$-glycan core, respectively. In sandwich-ELISAs, IgG-associated IgM, IgA, C1q, C3c and C-reactive protein (CRP) were detected as candidates for IgG immune complex constituents. We studied associations of the glycan of complexed $\mathrm{IgG}$ and disease activity according to the physician's global assessment of disease activity and the systemic lupus erythematosus disease activity index 2000 documented at the moment of blood taking. Our results showed significantly higher levels of Aleuria aurantia lectin and Lens culinaris agglutinin binding sites exposed on $\operatorname{IgG}$ complexes of patients with systemic lupus erythematosus than on those of normal healthy blood donors. Disease activity in systemic lupus erythematosus correlated with higher exposure of Aleuria aurantia lectin-reactive fucosyl residues by immobilized IgG complexes. Top levels of Aleuria aurantia lectin-reactivity were found in samples taken during the highest activity of systemic lupus erythematosus. Our results show that native circulating IgG complexes from active systemic lupus erythematosus patients expose fucosyl residues and their glycan core is accessible to soluble lectins. Two putative mechanisms may contribute to the increased exposure of these glycans: (1) the canonical N-glycosylation site of the $\mathrm{IgG}-\mathrm{CH} 2$ domain; (2) an IgG binding non-IgG molecule, like complement or C-reactive protein. In both cases the complexed IgG may be alternatively targeted to lectin receptors of effector cells, e.g. dendritic cells. Lupus (2015) 24, 569-581.

Key words: IgG; immune complex; glycosylation; lectin-ELISA; Aleura aurantia; Lens culinaris; SLEDAI; physicians global assessment

Correspondence to: Martin Herrmann, Department of Internal Medicine 3 and Institute for Clinical Immunology and Rheumatology, University of Erlangen-Nürnberg, Ulmenweg 18, 91054 Erlangen, Germany.

Email: Martin.Herrmann@UK-erlangen.de

${ }^{*}$ C.S. and J.Z. equally contributed. M.H. and L.E.M. equally contributed to senior authorship.

${ }^{\dagger}$ The present work was performed in fulfillment of the requirements for obtaining the degree 'Dr. med.' for J.Z.

Received 16 July 2014; accepted 3 October 2014

\section{Introduction}

IgG antibodies to self-antigens are hallmarks of chronic autoimmune diseases. High-affinity autoantibodies contribute directly to pathogenicity by multiple mechanisms. ${ }^{1}$ Systemic lupus erythematosus (SLE) constitutes an autoantibody-driven disease, characterized by multi-organ involvement with episodes of disease flares and remission 
over time. The current view is that the SLE pathogenesis relates to abnormal apoptosis and deficient elimination of apoptotic material, such as chromatin. This eventually leads to autoantibody production and formation of circulating and/or tissue-bound $\mathrm{IgG}$ immune complexes (IC). ${ }^{2}$ IgG autoantibody-binding to tissue-exposed autoantigens and/or insufficient receptor-mediated clearance of circulating IC (CIC) via the reticuloendothelial system may impair hepatic IC clearance and cause extra-hepatic IC deposition. ${ }^{3-5}$ Altered clearance of apoptotic chromatin via CIC formation results in enhanced inflammation and tissue damage. ${ }^{6,7}$

IgG is an example of a glycoprotein, characteristically carrying a complex $\mathrm{N}$-linked biantennary glycan, consisting of a core heptasaccharide structure with variable additions of fucose and outer arm sugar residues like galactose and sialic acids, attached to asparagine-297 of the heavy chain $\mathrm{CH} 2$ domain. 8,9 At least two dozen different Fc glycoforms, consisting of the heptasaccharide biantennary core with a combination of different numbers of core-fucose, galactose, bisecting N-acetyl glucosamine (GlcNAc) and terminal sialic acids, have been identified on polyclonal serum $\operatorname{IgG}^{10-12}$ and monoclonal IgG. ${ }^{13}$ Data from several studies indicate that these exposed glycans play important roles in structure and function of proteins; e.g. minor changes in a single glycan can affect protein folding and processing. ${ }^{4,15}$ In addition, the functionality of terminal sugars on IgG-N-glycans is of major biological importance. IgG without terminal galactose (G0) or core-fucose exhibit higher antibody dependent cell mediated cytotoxicity (ADCC). ${ }^{8,16-21}$. G0-IgG facilitates placental transport $^{22}$ and $\mathrm{IgG}$ without terminal sialic acid residues suppresses ADCC. $^{23}$ We employed the lectins Aleuria aurantia lectin (AAL) and Lens culinaris agglutinin (LCA) that bind to fucosyl residues and the fucosylated tri-mannose $\mathrm{N}$-glycan core, respectively, to assess the glycosyl residues exposed by complexed native $\mathrm{IgG}^{24}$ The target of our examination was not the isolated monomeric $\mathrm{IgG}$ molecule. Instead we investigated circulating $\mathrm{IgG}$ complexes, which are composed of a plethora of potentially glycosylated constituents. The latter are prone to partially redirect the IC from Fc receptors to the surface lectins of effector cells like monocytes, macrophages or dendritic cells.

The aims of the present study were to examine lectin targets associated with immobilized native complexed IgG in sera from patients with SLE. We analysed whether the exposure of fucosyl residues or the fucosylated tri-mannose $\mathrm{N}$-glycan core of complexed IgG is related to SLE disease activity and disease phenotype. A regional Swedish register containing well-characterized SLE patients with longitudinal serum samples formed the basis of this investigation.

\section{Material and methods}

\section{Subjects}

15 SLE patients (13 female, two male; mean age 40.0 years; range 18-64) were recruited to the study on the basis of an episode of raised disease activity over a period of time. All patients took part in a prospective control programme (clinical lupus register in northeastern Gothia) at the Rheumatology department at Linköping University hospital and met the validated 1982 American College of Rheumatology (ACR) as well as the 2012 Systemic Lupus International Collaborating Clinics classification criteria. ${ }^{25,26}$ The mean number of fulfilled ACR criteria was 5.7 (range 4-7). Serial serum samples were drawn at each visit to the rheumatologist during the study period (2-10 visits per individual; mean 6) and the sera were kept frozen at $-70^{\circ} \mathrm{C}$ until analysed. The physician's global assessment of disease activity (PGA; 0-4) and the SLE disease activity 2000 (SLEDAI) was recorded at each visit. ${ }^{27}$ All patients showed signs of disease activity defined as a SLEDAI peak score of at least 6 (mean 11.9; range 6-30) for a median time of 16.9 months (range 6-26 months). The terms increased disease activity referred to the time-point of highest and decreased disease activity referred to the timepoint of lowest SLEDAI score, respectively. Treatments with glucocorticoids and diseasemodifying anti-rheumatic drugs were recorded. Clinical characteristics are summarized in Table 1. Fifteen sex- and age-matched normal healthy blood donors (NHD) (13 female, 2 male; mean age 40.3 years) served as controls.

\section{Routine laboratory analyses}

Laboratory analyses at all visits included erythrocyte, leukocyte and platelet counts, urine albumin/ erythrocytes, creatinine, creatine kinase, antidoublestranded (ds) DNA antibodies, erythrocyte sedimentation rate, $\mathrm{C}$-reactive protein (CRP), complement protein $\mathrm{C} 3$ and $\mathrm{C} 4$, and classical haemolytic complement function. High sensitivity CRP (detection limit of $0.12 \mathrm{mg} / \mathrm{L}$ ) was analysed by turbidimetry at the clinical chemistry of the Linköping 
Table 1 Clinical characteristics of patients with SLE

\begin{tabular}{|c|c|c|c|c|c|c|c|}
\hline \multirow[b]{2}{*}{ Patient \# } & \multirow[b]{2}{*}{ Age/sex/ethnicity } & \multirow{2}{*}{$\begin{array}{l}\text { Recent onset } \\
\text { disease } \\
(\text { yes } / \text { no })\end{array}$} & \multirow[b]{2}{*}{ Manifestation at flare } & \multirow[b]{2}{*}{$\begin{array}{l}\text { Fulfilled } 1982 \\
\text { ACR criteria }\end{array}$} & \multirow{2}{*}{$\begin{array}{l}\text { Daily dosage } \\
\text { of Prednisolone } \\
\text { (at flare) }\end{array}$} & \multicolumn{2}{|c|}{ Other treatment at blood sampling } \\
\hline & & & & & & At flare & $\begin{array}{l}\text { Anytime during } \\
\text { the study }\end{array}$ \\
\hline 1 & 56/F/Caucasian & no & $\mathrm{CNS}$ & $3,5,6,11$ & 30 & Cyclo, Am & Rtx \\
\hline 2 & 18/F/Caucasian & no & Nephritis & $1,5,7,10,11$ & 7.5 & Am & $\mathrm{Am}$ \\
\hline 3 & 28/F/Caucasian & no & Serositis, leukopenia & $4,5,6,7,9,10,11$ & 20 & Rtx & Aza, Am \\
\hline 4 & 46/F/Asian & no & $\begin{array}{l}\text { Fever, rash, } \\
\text { oral ulcers, } \\
\text { CNS, leukopenia }\end{array}$ & $1,3,4,7,8,9,11$ & 50 & $\mathrm{Mmf}$ & Rtx, Mmf \\
\hline 5 & 36/F/Caucasian & no & Vasculitis, arthritis, rash & $1,5,6,7,9,10,11$ & 15 & Rtx & Aza, Am \\
\hline 6 & 39/F/Caucasian & no & Nephritis, arthritis & $1,3,5,7,10,11$ & 2.5 & $\mathrm{Am}$ & $\mathrm{Am}$ \\
\hline 7 & 64/F/Caucasian & no & Nephritis, vasculitis, rash & $1,3,5,7,9,10,11$ & 30 & Rtx, Am & $\mathrm{Am}$ \\
\hline 8 & 37/M/Caucasian & yes & Serositis & $6,9,10,11$ & 20 & $\mathrm{Am}$ & $\mathrm{Am}$ \\
\hline 9 & 33/F/Caucasian & yes & Nephritis, leukopenia & $7,9,10,11$ & 30 & $\mathrm{Am}$ & Siro, Am \\
\hline 10 & 54/M/Caucasian & no & $\begin{array}{l}\text { Nephritis, leukopenia, } \\
\text { rash, myositis, } \\
\text { leukopenia }\end{array}$ & $1,5,6,7,9,10,11$ & 80 & $\mathrm{Mmf}$ & $\mathrm{Mmf}$ \\
\hline 11 & 21/F/Asian & yes & $\begin{array}{l}\text { Nephritis, leukopenia, } \\
\text { rash, arthritis }\end{array}$ & $1,5,7,9,10,11$ & 15 & Cyclo, Am & Mmf, Am \\
\hline 12 & 30/F/Caucasian & yes & $\begin{array}{l}\text { Serositis, rash, } \\
\quad \text { thrombocytopenia }\end{array}$ & $3,5,6,9,11$ & 30 & Aza, Am & Aza, Am \\
\hline 13 & 60/F/Caucasian & no & Serositis & $5,6,9,10,11$ & 40 & $\mathrm{Mmf}$ & $\begin{array}{l}\text { Aza, Mmf, } \\
\text { Mtx, Bmab }\end{array}$ \\
\hline 14 & 31/F/Caucasian & yes & Nephritis, rash & $1,3,5,6,7,10,11$ & 60 & Cyclo, Am & $\mathrm{Mmf}, \mathrm{Am}$ \\
\hline 15 & 49/F/Caucasian & yes & $\begin{array}{l}\text { Vasculitis, } \\
\text { oral ulcers, headache }\end{array}$ & $3,4,10,11$ & 30 & Aza & $\mathrm{Am}$ \\
\hline
\end{tabular}

Am: antimalarials; Aza: azathioprine; Bmab: belimumab; Cyclo: cyclophosphamide; Mmf: mycophenolate mofetil; Mtx: methotrexate; Rtx: rituximab; Siro: sirolimus.

University Hospital. Complement analyses were performed at the University Hospitals in Uppsala or Linköping, Sweden. Microscope slides with fixed Crithidia luciliae (ImmunoConcepts) were used to analyse IgG class anti-dsDNA antibodies by indirect immunofluorescence microscopy (cut-off titre 1:10, corresponding to $>99$ th percentile among healthy female blood donors). Anti-dsDNA antibody levels were end-point titration in two-fold dilution steps.

Analysis of CIC composition by IgG capture ELISA and determination of exposed IgG glycosyl residues by IgG capture lectin ELISA

To analyse the circulating native complexed IgG, an IgG capture ELISA was performed. Ninety-six well microtitre plates (Nunc, F96 MaxiSorp) were coated with $2 \mu \mathrm{g} / \mathrm{ml}$ anti-human IgG $\mathrm{F}(\mathrm{ab})_{2}$-fragment $\left(\mathrm{F}(\mathrm{ab})_{2}\right.$ goat anti-human serum IgG, $\mathrm{H}+\mathrm{L}$ chain, Jackson Laboratories Immunoresearch) in coating buffer $(0.1 \mathrm{M}$ $\left.\mathrm{Na}_{2} \mathrm{CO}_{3}, \mathrm{NaHCO}_{3}\right)$ pH 9.6 at $4{ }^{\circ} \mathrm{C}$ overnight. Plates were blocked with 3\% bovine serum albumin (Sigma-Aldrich) in phosphate buffered saline (PBS) containing $0.05 \%$ tween-20 (Roth) at $37^{\circ} \mathrm{C}$ for 2 hours. After blocking, patient sera or sera from normal healthy donors were diluted $1: 1000$ in PBS-tween and incubated at $37^{\circ} \mathrm{C}$ for 2 hours. These serum dilutions resulted in a saturating binding of IgG to the ELISA plate. After every incubation step the plates were washed three times with $200 \mu \mathrm{l}$ PBS-tween. Horseradish-peroxidase (HRP)labelled anti-human IgG-antibody (Southern Biotech), anti-human IgA-antibody (Southern Biotech), anti-human IgM antibody (Southern Biotech), anti-human Clq (Abcam), anti-human C3c (Abcam) or anti-human CRP (Abcam) in the concentration recommended by manufacturer were used for the detection of $\mathrm{IgG}$ and the other components of complexed native $\mathrm{IgG}$, respectively.

In order to analyse the exposure of specific glycans of the glycoprotein $\mathrm{IgG}$ in the sera, an $\operatorname{IgG}$ capture lectin ELISA was performed (Figure 1(a)). After coating 96 well plates with the anti-human IgG $\mathrm{F}(\mathrm{ab})_{2}$-fragment described above, plates were blocked with deglycosylated blocking buffer $(3 \%$ gelatine, $\quad 0.1 \% \quad \mathrm{CaCl}_{2}, \quad 0.1 \% \quad \mathrm{MgCl}_{2}, \quad 0.05 \%$ tween-20) at $37^{\circ} \mathrm{C}$ for 2 hours. This buffer was pretreated for 24 hours with $1 \%$ periodic acid (Merck) to destroy glycosylation sites of the gelatine. The $\mathrm{pH}$ was restored by dialysis against tris buffered 


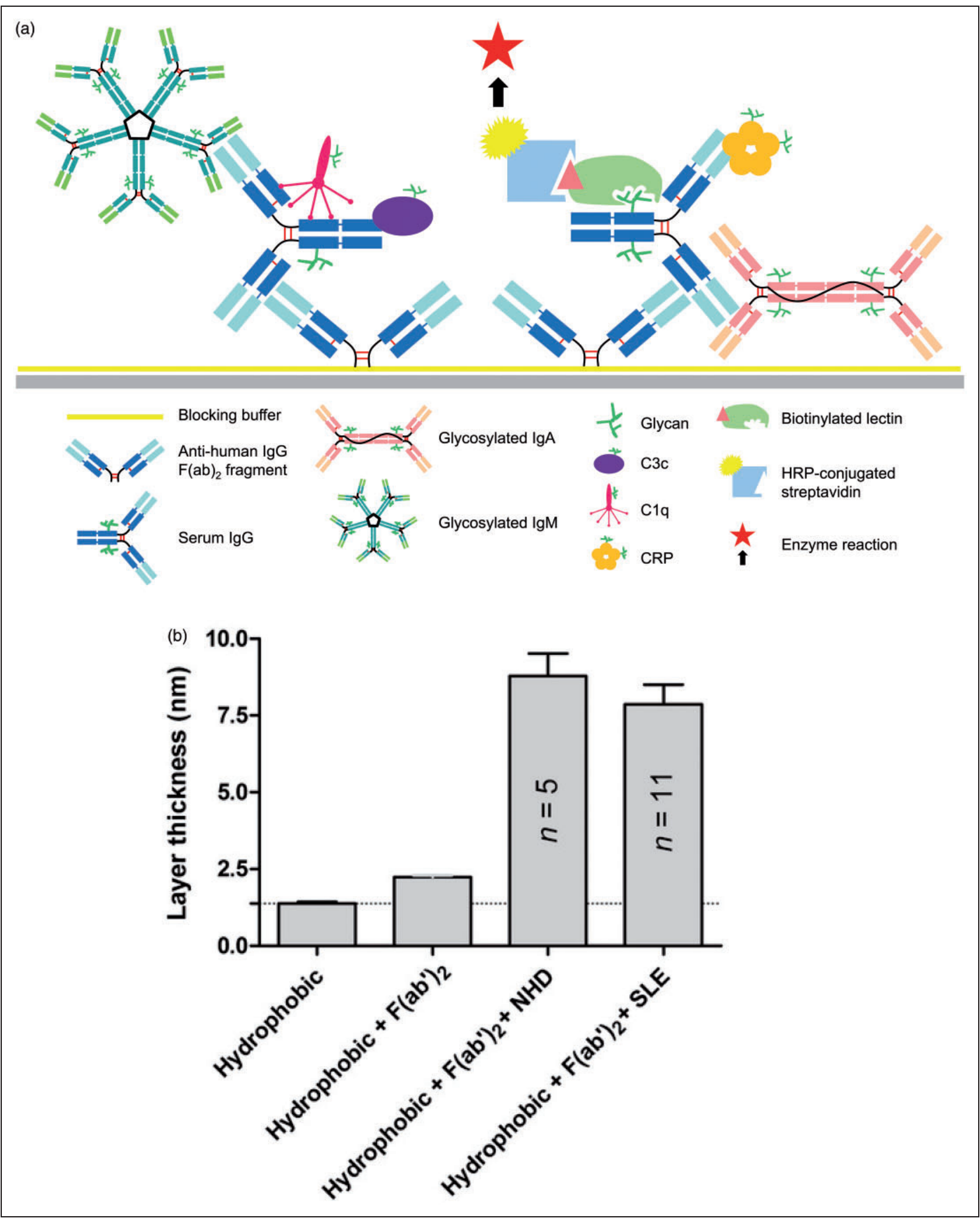

Figure 1 Lectin-ELISA of serum IgG and adsorption of serum proteins to F(ab') ${ }_{2}$-fragments.

In order to detect carbohydrate variations of complexed IgG from serum of patients with SLE we performed Lectin-ELISAs. The plates were coated with goat anti-human $\operatorname{IgG} \mathrm{F}(\mathrm{ab})_{2}$-fragment, treated with deglycosylated blocking buffer to avoid loading unspecific glycans onto the plates. After incubation with serum dilutions and several washing steps biotinylated lectins were employed to detect fucosyl residues and fucosylated tri-mannose $\mathrm{N}$-glycan core sites of the complexed native IgG molecules (a). Ellipsometry was used to measure layer thickness of the proteins bound to the anti-human $\operatorname{IgG} F(\text { ab') })_{2}$-fragment. The hydrophobic surface constitutes the background. At least $1 \mathrm{~nm}$ of $\mathrm{F}(\mathrm{ab})_{2}$-fragments bound to the surface (mean value of 10 measurements shown). IgG from sera of five NHD and 11 SLE patients was immobilized to a similar extent of 5-7 nm; the data represent mean values of 10 separate measurements of each individual serum sample (b). 
saline containing $0.1 \% \mathrm{CaCl}_{2}, 0.1 \% \mathrm{MgCl}_{2}$ (TBS$\mathrm{Ca}-\mathrm{Mg}$ ) until a $\mathrm{pH}$-value of 7.4 was reached. After blocking, patient sera or sera from normal healthy donors were diluted 1:1000 in TBS-Ca-Mg and $0.05 \%$ tween-20 and incubated at $37^{\circ} \mathrm{C}$ for 2 hours. These serum dilutions resulted in a saturating binding of $\mathrm{IgG}$ to the ELISA plate. After every incubation step the plates were washed three times with $200 \mu \mathrm{l}$ TBS-Ca-Mg-tween. Fifty ng/ml biotinlabelled AAL or LCA (Vector Laboratories) were used to detect glycosyl residues exposed by the captured IgG complexes and incubated at room temperature for 1 hour. After washing, plates were incubated with HRP-streptavidin (Jackson Laboratories Immunoresearch) at the recommended concentration for 1 hour at room temperature.

Detection was performed for both types of ELISA with the addition of substrate solution $\left(0.1 \mathrm{M} \mathrm{Na}_{2} \mathrm{HPO}_{4}, 0.05 \mathrm{M}\right.$ citrate acid monohydrate, $0.02 \% \quad \mathrm{H}_{2} \mathrm{O}_{2}$ and $100 \mu \mathrm{g} / \mathrm{ml}$ Tetra-methyl benzidine, $\mathrm{pH}=5$, Merck) for 10 minutes and the colour reaction was stopped with $25 \%$ sulphuric acid (Merck). Optical density values were obtained with the ELISA-reader (Tecan infinite F200 Pro) employing a $450 \mathrm{~nm} / 620 \mathrm{~nm}$ filter/reference pair.

\section{Ellipsometry}

Ellipsometry is a highly sensitive optical method that has been used since the 1960s to determine the thickness of an adsorbed bioorganic layer with a practical resolution of about $0.1 \mathrm{~nm} .^{28}$ Thus, we used ellipsometry to confirm the adsorption of $\mathrm{F}(\mathrm{ab})_{2}$-fragments and serum IgG onto surfaces of methylated (hydrophobic) silicon as previously described. ${ }^{29,30}$ Measurements were performed on: (1) bare methylated surface; (2) with $\mathrm{F}(\mathrm{ab})_{2}$-fragments adsorbed; (3) with sera adsorbed to $\mathrm{F}(\mathrm{ab})_{2}$-fragments.

\section{Ethics}

Oral and written informed consent was obtained from all subjects. The study protocol was approved by the Linköping University Ethical Review Board (M75-08/2008).

\section{Results}

Complexed IgG from patients with SLE expose fucose residues

Employing ellipsometry, we confirmed the adsorption of $\mathrm{F}(\mathrm{ab})_{2}$ of more than $1 \mathrm{~nm}$ onto the
Table 2 Adsorption of serum proteins to immobilized serum native $\mathrm{IgG}$

\begin{tabular}{llll}
\hline & $\begin{array}{l}\text { NHD } \\
(\text { Mean } \pm S D)\end{array}$ & $\begin{array}{l}\text { SLE } \\
(\text { mean } \pm 1 D)\end{array}$ & $\begin{array}{l}\mathrm{t} \text {-test } \\
\text { (p value) }\end{array}$ \\
\hline IgM (OD 450 nm) & $0.27 \pm 0.096$ & $0.29 \pm 0.093$ & 0.30 \\
IgA (OD 450 nm) & $0.36 \pm 0.079$ & $0.4 \pm 0.059$ & 0.10 \\
C1q (OD 450 nm) & $0.05 \pm 0.007$ & $0.06 \pm 0.041$ & 0.07 \\
C3c (OD 450 nm) & $0.07 \pm 0.01$ & $0.09 \pm 0.04$ & 0.000044 \\
CRP (OD 450 nm) & $0.04 \pm 0.005$ & $0.05 \pm 0.02$ & 0.25 \\
\hline
\end{tabular}

hydrophobic surface and this layer immobilized similar levels of serum proteins from patients and controls (Figure 1(b)). Once the immobilization of random serum IgG complexes was confirmed, their exposure of AAL and LCA binding glycoepitopes in consecutive serum samples of 15 patients with SLE and in sera of 15 NHD were measured employing a lectin-ELISA. We aimed to create a profile of the surface structure of IgG from patients with SLE using detection antibodies against IgM, IgA, C1q, C3c and CRP. The analysis confirmed equal and saturating IgG-coating by sera from patients with SLE and NHD. Table 2 shows that secondary antibodies against IgM, IgA, complement component C1q and CRP showed no significant difference between patients with SLE and the controls. However, we observed a significantly higher reactivity of the complexed $\mathrm{IgG}$ with the anti-C3c antibody ( $p=0.000044)$ from the sera of patients with SLE.

Figure 2 shows that the exposure of glycosyl residues was independent of the age of the patients (analysed at the first visit) or the controls. The exposure of glycan sites by complexed random IgG in the sera of patients with SLE varied during the course of the disease and is represented in Figure 3 as black circles. In general, the complexed IgG showed a higher binding of AAL (Figure 3(a), $p<0.00001$ ) and LCA (Figure 3(b), $p<0.05)$ than NHD samples.

Exposure of fucosyl residues on $\operatorname{Ig} G$ is associated with high SLE disease activity and low serum C3 levels

We also noticed that disease activity in SLE, demonstrated by PGA scores at each visit, was associated with higher levels of AAL reactivity (Figure 4(a)). It was observed that during times of increased disease activity (PGA 1-3) patients with SLE display significantly increased AAL reactivity than NHD $(p=0.000000844)$. Interestingly, SLE patients clinically judged as non-active $(\mathrm{PGA}=0$, 


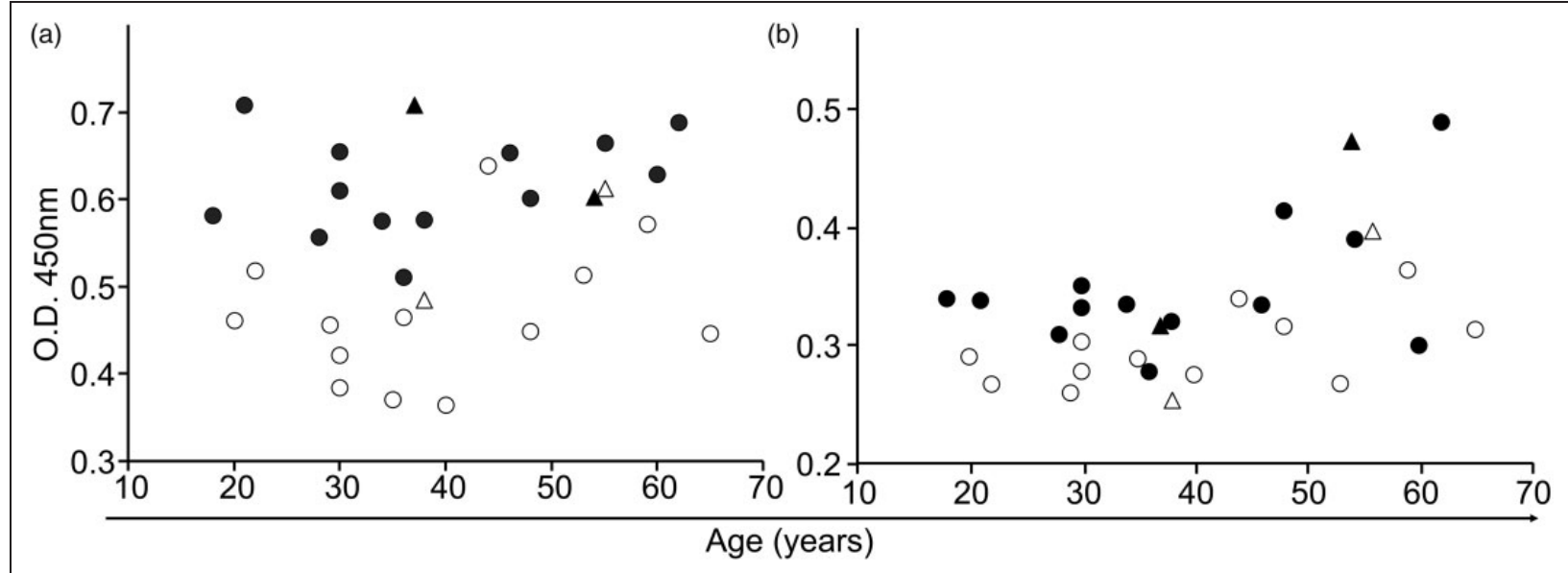

Figure 2 Binding of AAL and LCA to immobilized native IgG.

Lectin ELISA in sera from patients with SLE (close symbols) and normal healthy donors (open symbols). Binding of AAL and LCA to immobilized complexed $\mathrm{IgG}$ is shown in (a) and (b), respectively. Using all serum samples of the first visit of each patient no association among lectin binding, age and sex (female circles, male triangles) was to be observed.

i.e. remission) still showed higher AAL binding to complexed serum IgG than NHD $(p=0.0038)$. When comparing patients in remission $(\mathrm{PGA}=0)$ with mild $(\mathrm{PGA}=1)$, moderate $(\mathrm{PGA}=2)$ and high $(\mathrm{PGA}=3)$ disease activity, we found a significant increase of AAL-reactivity $(p=0.0007,0.0011$ and 0.016 , respectively). The association of raised disease activity and higher degree of fucosyl accessibility of native complexed $\mathrm{IgG}$ was also demonstrated applying the SLEDAI (Figure 4(b)). Patients with inactive SLE (SLEDAI =0) exhibit significantly higher AAL reactivity in comparison with NHD $(p=0.0011)$. Patients with SLE and SLEDAI scores higher than 0 show significantly higher levels of AAL binding than NHD $(p=0.0000007)$ and patients with SLE and a SLEDAI score of $0(p=0.0002)$.

We calculate the positive and negative predictive values (PPV and NPV respectively) of fucosyl residues exposed by random IgG and disease activity measured by SLEDAI and PGA, respectively. Samples with SLEDAI higher than 5 and PGA scores higher than 0 were considered as having increased disease activity. Positive values for AAL reactivity were considered as higher than $90 \%$ of the NHD. Considering SLEDAI as gold standard, the PPV was $70.3 \%$, the NPV $69.8 \%$, sensitivity $61.9 \%$ and specificity $77.1 \%$ for the detection of fucosyl residues in IgG IC by AAL. Similarly, for PGA-score we calculated PPV 89.2\%, NPV 54.7\%, sensitivity $57.9 \%$ and specificity $87.9 \%$.

Correlation analysis revealed a strong negative association of the exposure of fucosyl residues with the levels of circulating complement component C3 $(p<0.0001$; Figure 4(c)). Other laboratory parameters $(\mathrm{C} 4$, erythrocyte sedimentation rate, haemoglobin, leukocyte and thrombocyte counts) did not show relevant associations with the exposure of fucosyl residues on the immobilized IC.

Increased disease activity associates with increased exposure of $A A L$-binding sites in individual patients

We observed an association between the highest AAL reactivity of complexed random $\operatorname{IgG}$ of a patient and increased disease activity for the majority of the patients studied. In our follow-up cohort of 15 patients with SLE, $11(73 \%)$ showed maximum levels of AAL-binding of complexed IgG during increased disease activity (Figure 5(a)). Using the PGA to quantify disease activity we noticed a similar outcome in seven out of 15 $(47 \%)$ patients. Interestingly, it turned out that five patients with SLE showed no difference in PGA score while they reached the maximum and minimum levels of AAL-binding (Figure 5(b)). In order to closely observe the association between exposure of fucosyl residues by native IgG complexes and disease activity we illustrated the courses of AAL-reactivity and SLEDAI in the same plot (Figure 6). We included in this figure all SLE patients with at least five available longitudinal serum samples and a peak of SLEDAI between the first and the last determined SLEDAI scores (patient numbers 1-7, 9, 10, 12, 13). Eight from these 11 patients (numbers 2, 3, 4, 6, 9, 10, $12,13)$ showed similarities between SLEDAI and 


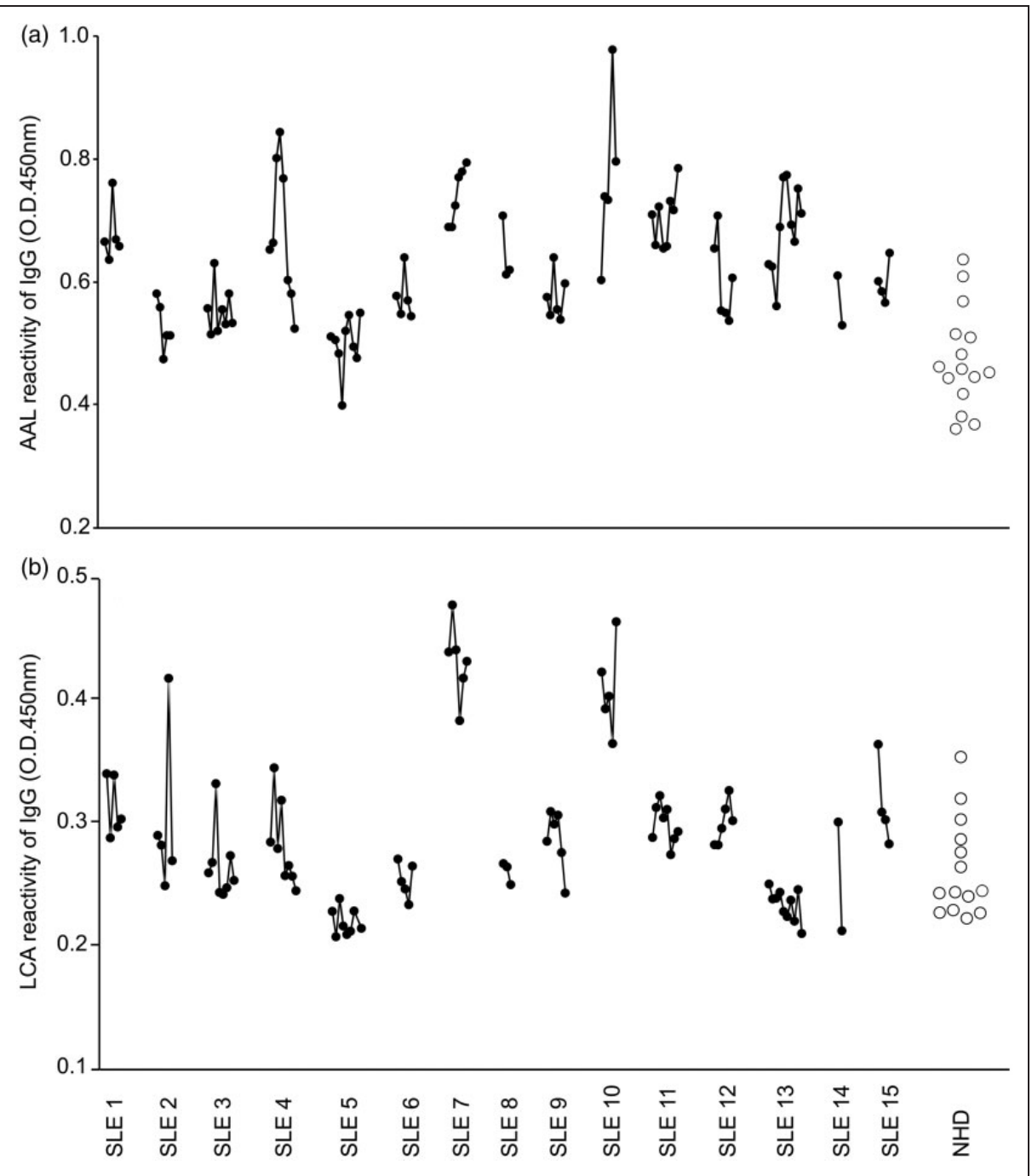

Figure 3 Binding of AAL or LCA to complexed IgG molecules in consecutive serum samples from patients with SLE. (a) AAL-reactivity of IgG in consecutive serum samples from patients with SLE and sex- and age-matched controls. IgG from patients with SLE show higher exposure of fucosyl residues during the course of disease than $\mathrm{NHD}\left(p=8.84641 \times 10^{-06}\right)$. (b) LCA-reactive IgG in consecutive serum samples from patients with SLE and sex- and age-matched controls. Exposure of fucosylated tri-mannose $\mathrm{N}$-glycan core sites by $\mathrm{IgG}$ from serum samples of patients with SLE is found at higher levels than in samples from sex- and age-matched NHD $(p=0.02)$.

AAL-reactivity, although the absolute levels were rather different between the individual patients.

\section{Discussion}

SLE is a disease of multifactorial genesis, in which immune complex formation by autoantibodies, complement activation and cytokine production lead to persistent inflammation and subsequent tissue damage in various organs. ${ }^{31-33}$ In the pathogenesis of SLE, the impaired and/or delayed clearance of apoptotic cells is discussed as a reason for the development of nuclear autoantibodies. ${ }^{34,35}$
Enzymatic removal of the glycan structure of complexed Ig with endoglycosidase $\mathrm{S}$ has been shown to diminish various pro-inflammatory features of these CICs in-autoimmune conditions. ${ }^{36}$ Activation of the complement system by classical pathway, induction of interferon- $\alpha$ production and/ or modulation of immune responses by binding to Fc $\gamma$ receptors are the putative pathogenic mechanisms. This suggests the assumption that the molecular structures of CICs, which include their glycans strongly impact their functionality and thus their inflammation-driving potential. ${ }^{31}$ In rheumatoid arthritis (RA), a further chronic inflammatory disease, several studies have revealed that 

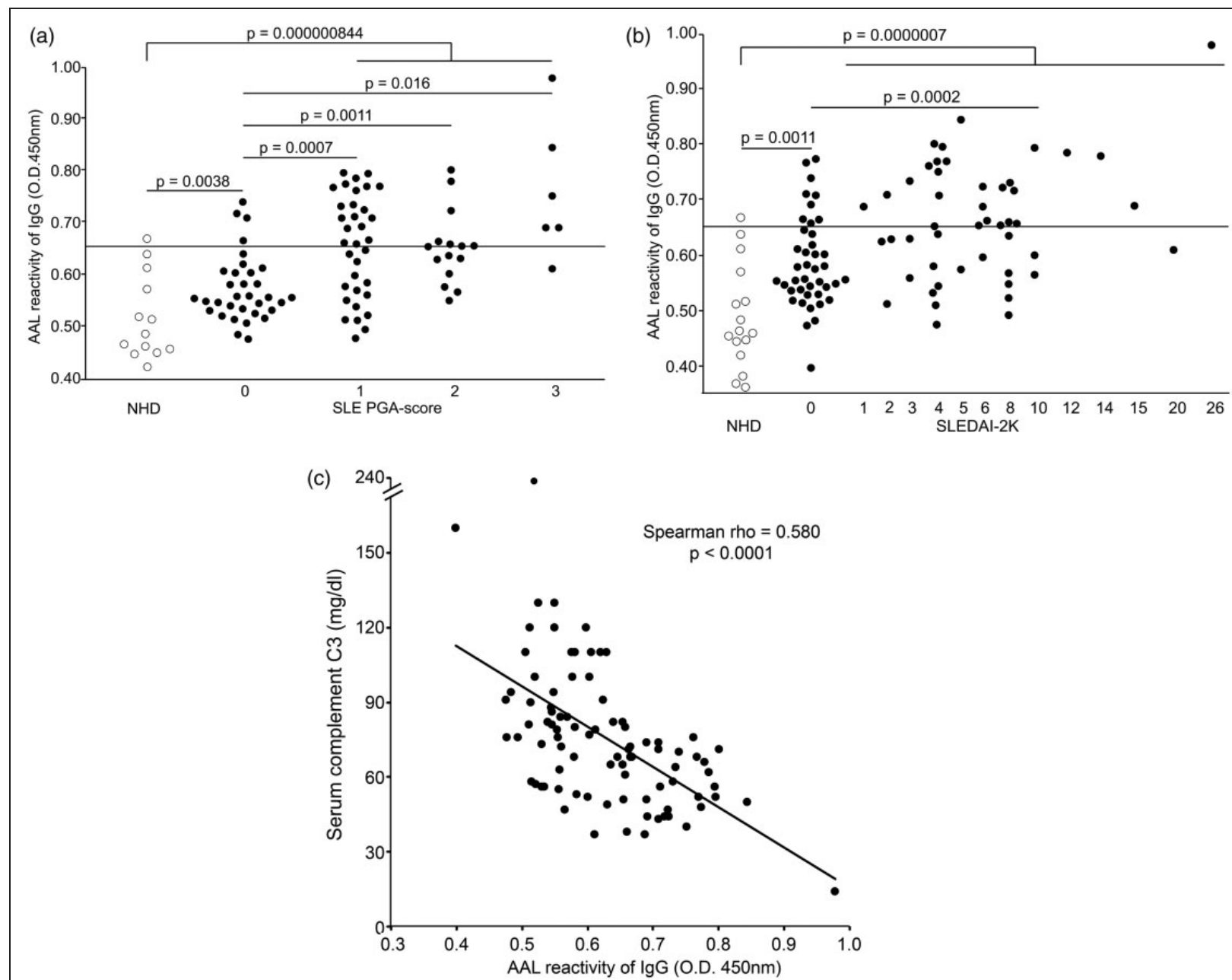

Figure 4 Association between exposure by native complexed IgG of AAL binding sites from patients with SLE, disease activity and low serum $\mathrm{C} 3$.

(a) Association between AAL reactivity of complexed IgG from patients with SLE and PGA. During times of raised disease activity (PGA 1-3) patients with SLE exhibit higher levels of AAL binding serum IgG. Patients with SLE and a PGA score of 0 show significantly higher AAL reactive $\operatorname{IgG}$ than NHD $(p=0.0038)$. In comparison to patients with SLE and a PGA score of 0 SLE patients with PGA score of $1(p=0.0007)$, a PGA score of $2(p=0.0011)$ or a PGA score of $3(p=0.016)$ also present significantly higher levels of AAL reactivity. Patients with a PGA score of 1, 2 and 3 when compared to NHD ( $p=0.00000084$; not shown). (b) Systemic Lupus Erythematosus Disease Activity Index-2K (SLEDAI), AAL reactivity of serum IgG from patients with SLE turns out to be strikingly elevated in times of increased disease activity (SLEDAI $>0$ ). Serum samples from patients with SLE and a SLEDAI score of 0 present significantly higher amounts of AAL binding IgG than samples from NHD ( $p=0.0011)$. Patients with SLE and raised SLEDAI $>0$ show significantly higher levels of IgG reactive with AAL in comparison to patients with a SLEDAI score of $0(p=0.0002)$ and to NHD $(p=0.0000007)$. (c) Spearman rho correlation analysis. The strong inverse association between AAL reactivity of random complexed IgG and serum complement C3 levels is shown $(r=-0.580 ; p<0.0001)$. Horizontal lines in (a) and (b) represent the cut-off for AAL-reactivity (percentile 90 of the NHD cohort).

galactosylation of serum IgG correlates with disease activity and can be influenced by various types of therapy. ${ }^{37}$ The fucosylation of denatured IgG heavy chains was significantly increased in RA as measured by lectin binding. ${ }^{38}$ Furthermore, there is a positive correlation between galactosylation and sialylation of $\operatorname{IgG}$ with the improvement of RA during pregnancies and post-partum flares. ${ }^{39}$
Glycans are potential ligands for a plethora of lectin receptors found on immune effector cells, which are used as phagocytic receptors. Data about IgG glycosylation status and glycan exposure of native IgG in SLE during disease activity and remission are still scarce. In this study, we analysed serum samples of 15 well-characterized Swedish SLE patients. The samples were drawn at 


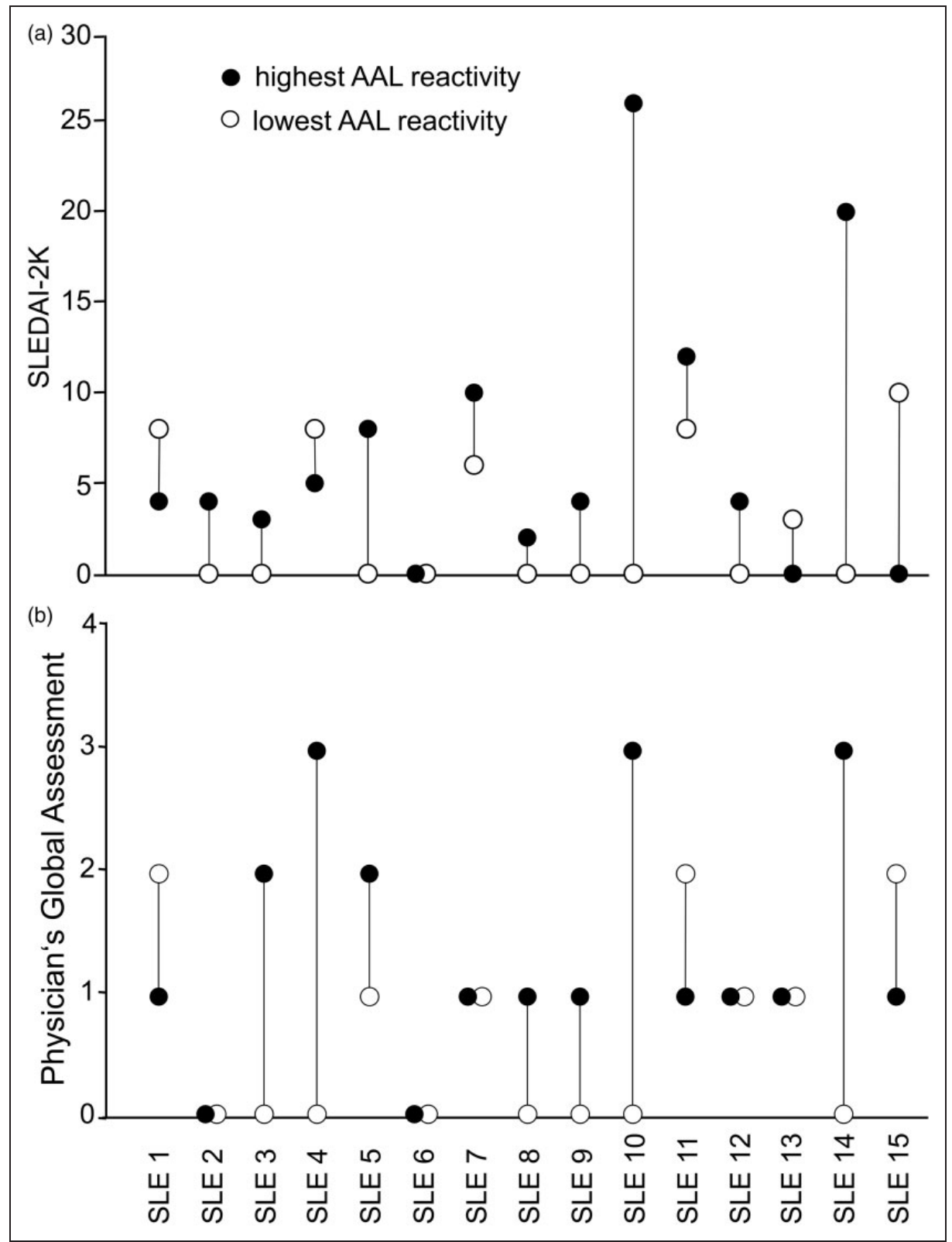

Figure 5 Association between maximum and minimum AAL reactivity and clinical activity.

(a) Immobilized complexed IgG of patients with SLE display increased AAL binding during times of increased disease activity (SLEDAI). Four patients exhibit a higher SLEDAI score during lowest detectable AAL binding of serum IgG. Eleven patients $(73 \%)$ present a higher SLEDAI score at the time point of the highest AAL binding. (b) PGA also demonstrates that IgG complexes of patients with SLE display higher levels of AAL binding during increased disease activity. For five patients with SLE there is no difference in PGA score for the time-points of highest and lowest detectable AAL reactivity. Three patients show a higher PGA score during time of lowest AAL binding of random serum IgG than during highest accessibility of fucosyl residues. Seven patients with SLE exhibit a higher PGA score at the time of the highest exposure of fucosylated sites of native IgG complexes.

consecutive visits during active and non-active periods of disease. The grade of disease activity was quantified for every sample with PGA and SLEDAI. The serum samples of 15 sex- and agematched healthy blood donors served as controls.
In Lectin-ELISAs the binding of AAL and LCA to immobilized native serum IgG was quantified to detect exposure of fucosyl residues and of the fucosylated tri-mannose $\mathrm{N}$-glycan core, respectively. For Lectin-ELISAs it was necessary to prepare a 


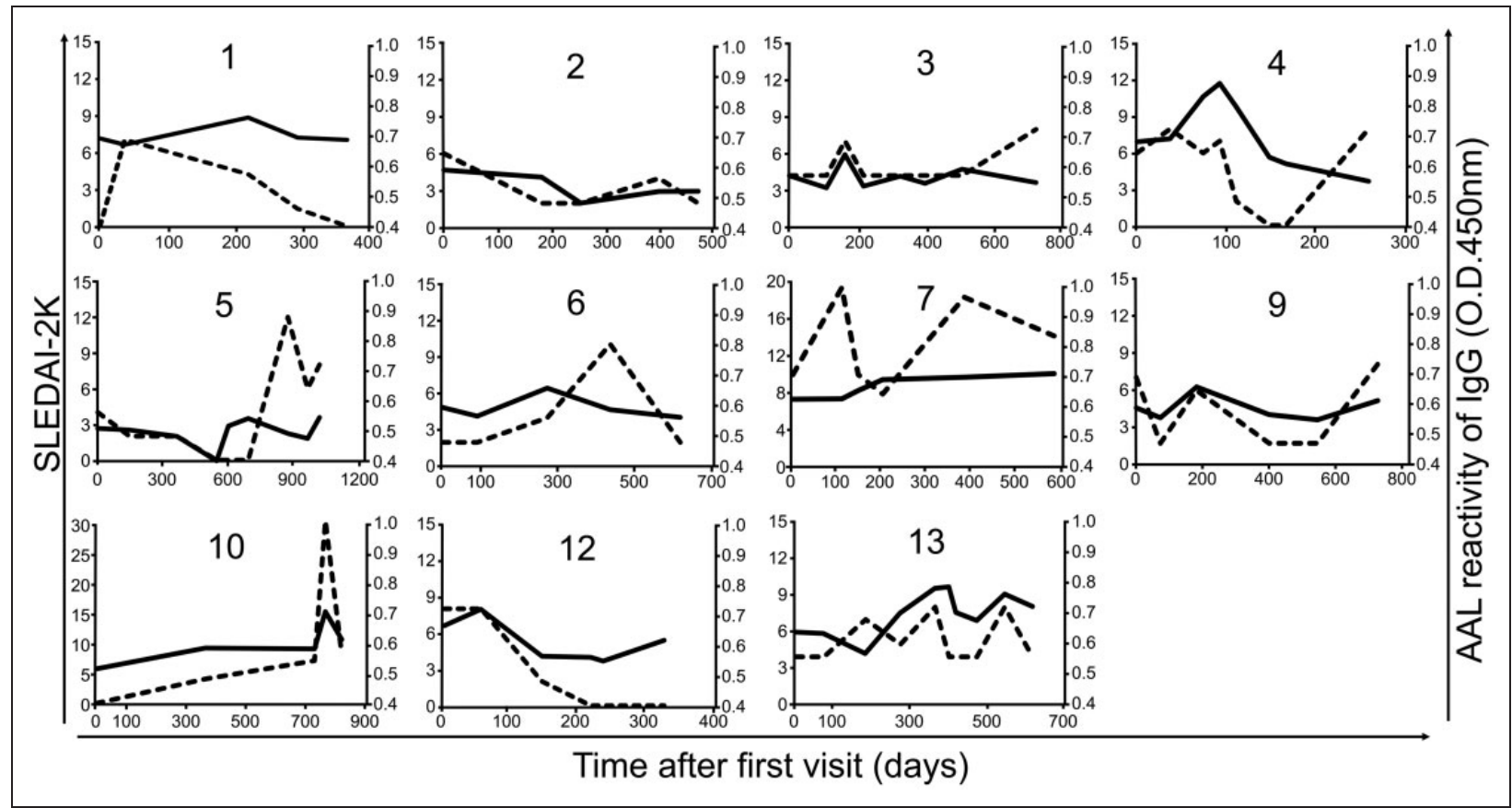

Figure 6 Exposure of fucosyl residues by random IgG during the course of disease.

Association between Systemic Lupus Erythematosus Disease Activity Index-2K (SLEDAI-2K) (broken line) and AAL reactivity during the course of disease (solid line). In this figure the course of disease activity (raised SLEDAI-2K) and the course of AAL reactive serum IgG is shown in order to detect parallels. All patients with at least five consecutive serum samples and a SLEDAI-2K peak between the first and the last collected SLEDAI-2K score are included (patient numbers 1-7, 9-10, 12-13).

special deglycosylated blocking buffer (treated with $1 \%$ periodic acid) to avoid loading unspecific glycan structures onto the polystyrene plates during the process of blocking.

Since several immune cells employ glycan-lectin recognition patterns, ${ }^{40}$ we aimed not to analyse the glycan of isolated and denatured $\mathrm{IgG}$ in the peripheral blood, but the glycans exposed by circulating native IgG complexes. We argue that a complex can be phagocytosed in a lectin dependent manner if any of the components exposes a corresponding glyco-epitope ${ }^{41}$. This may even apply to the bound antigen. ${ }^{42}$ In contrast, internal glycans are going to be ignored when they are inaccessible due to steric hindrance. However, the latter are usually detected with techniques that require the denaturation of the target, like lectin blot or mass spectrometry. ${ }^{12,43,44}$

Thus, we determined the level of immobilized native $\mathrm{IgG}$, representing loading control, as well as the level of the IgG-associated candidate molecules IgA, IgM, C3c, C1q and CRP. This selection is by far not complete, but represents a couple of molecules that are reportedly found in circulating IgG complexes. The level of IgG-bound complement proteins correlates with its consumption. In these types of ELISA, we analysed native serum
IgG complexes under preservation of their molecular integrity and thus tried to gain new information about the in vivo function of various glycosyl residues exposed by complexed, circulating immunoglobulins.

The analysis revealed that only the $\mathrm{C} 3 \mathrm{c}$ epitope was significantly increased in patients with SLE. Since C3c is a stable cleavage product of C3 covalently bound to target structures, the detection of $\mathrm{C} 3 \mathrm{c}$ gives a line on $\mathrm{C} 3$ metabolism. It is well-known that SLE is associated with an elevation of C3 turnover even during times of remission as a feature of chronic inflammation, whereby an upcoming exacerbation can be identified by a decline of $\mathrm{C} 3$, reflecting massive $\mathrm{C} 3$ consumption. ${ }^{45}$ The exposure of fucosyl residues on circulating IgG IC correlated highly significant with the consumption of serum complement $\mathrm{C} 3$. However, there was no association between $\mathrm{C} 3 \mathrm{c}$ detected on IgG IC and AAL reactivity (data not shown). This suggests that the consumption of $\mathrm{C} 3$ may rather be associated to the deposition of IC in tissues and therefore the measured AAL reactivity may reflect indirectly an ongoing immune complex deposition.

Employing the lectins as detection tool, we observed a higher exposure of IgG fucosyl residues both in sera of patients with SLE in general, and 
even more at time-points of increased disease activity. Figure 6 shows the marked parallels of increased accessibility of fucosylated sites of random IgG complexes and disease activity of some patients, giving a hint that there is an association between these two parameters. It has been shown in several studies that patients with RA display IgG with low galactosylation after flares, when compared to those in remission. The latter show levels of galactosylation similar to NHD. Fucosylation of IgG, conversely, turned out to be significantly increased throughout the whole course of RA. ${ }^{43}$ Under- and non-fucosylation of serum $\mathrm{IgG}$ is known to elevate the binding affinity to the Fc $\gamma$ RIIIa and thus to enhance its proinflammatory activity. ${ }^{12,44}$ Former studies revealed that the binding affinity of defucosylated IgG to the Fc $\gamma$ RIIIa might be 10-fold higher than that of immunoglobulins without decreased fucosylation. ${ }^{46}$ However, these results were obtained by employing genetic modification of host cells and denaturing conditions like treatment at $85^{\circ} \mathrm{C}$ to $100^{\circ} \mathrm{C}$, mass spectrometry or electrophoretic separation of $\mathrm{IgG}$ heavy and light chains. Consequently, these techniques are prone to additionally detect glycan structures hidden in the native molecules. Applying these methods functional characteristics of the native $\mathrm{IgG}$ complexes are lost.

Our investigation revealed that patients with SLE exhibit a significantly higher exposure of fucosyl residues of complexed serum IgG both in times of decreased and even more during times of increased disease activity.

There are several possibilities that could explain the increased binding of AAL to circulating complexed IgG: (1) increased fucosylation; (2) increased exposure of fucosyl residues on random $\mathrm{IgG}$; (3) a fucosylated glycan bound to the variable region of certain IgG molecules; (4) fucosyl residues exposed by the IgG associated molecules. Employing lectinELISA, we cannot distinguish which one of these mechanisms, if any, is responsible for our observations. Nevertheless, recognition of IC by immune cells may not distinguish the exact localization of the interacting fucosyl residues but just the presence of a glyco-epitope. Complexed $\mathrm{IgG}$ molecules exposing highly fucosylated sites may favour their uptake by binding to phagocyte surface lectin receptors and thus drive chronic inflammatory diseases like SLE and possibly others. After evaluating the performance of the test predicting periods of high disease activity we can consider the analysis of fucosylated sites exposed by circulating complexed IgG as an appropriate laboratory parameter to monitor patients with chronic inflammatory rheumatic diseases.

Besides the markedly elevated level of exposed fucosyl residues, we also detected a significant increase of LCA binding, suggesting more fucosylated tri-mannose $\mathrm{N}$-glycan core sites in the complexed IgG of patients with SLE. It had been reported that highly mannosylated IgG strongly activate Fc $\gamma$ RIIIa receptors and ADCC, ${ }^{44,47}$ fostering pro-inflammatory immune responses. But since those over-mannosylated IgGs also show reduced core fucose, it is not known which of these two features of glycosylation was responsible for the effect. Furthermore, highly mannosylated immunoglobulins exhibit increased affinity to the acute phase protein mannose-binding lectin (MBL), which usually binds to carbohydrate structures on the surface of pathogens and consecutively activates the complement system (lectin pathway). ${ }^{44}$ As a result, highly mannosylated immunoglobulins and microparticles display an increased rate of clearance. ${ }^{41,44,48}$ MBL comes along with higher susceptibility for SLE and it is presumed that MBL might foster the clearance of CIC, ${ }^{49}$ suspected to play an important role for the development of tissue damage in SLE. A possible explanation for the increased exposure of fucosylated tri-mannose $\mathrm{N}$-glycan core sites by native $\mathrm{IgG}$ is that $\mathrm{IgG}$ with higher accessibility to mannosylated sites is synthesized in SLE to act protectively by raising the affinity between CICs and MBL and thus fostering accelerated clearance of potentially tissue damaging IC.

\section{Funding}

This work was supported by the German Research Foundation (DFG) SFB 643 (project B5) and a training grant IRTG 643 to $\mathrm{MH}$ and by the County Council of Östergötland, the Swedish Society for Medical Research, the Swedish Rheumatism Association, the Swedish Society of Medicine, the Professor Nanna Svartz foundation and the King Gustaf V 80-year foundation to CS. IM and RB were supported by WUBMRC, NAS of Ukraine and State Fund for Fundamental Researches of Ukraine (project GP/ F49/169).

\section{Conflict of interest statement}

The authors have no conflicts of interest to declare. 


\section{Acknowledgements}

The authors thank research nurse Marianne Peterson, the clinicians at Linköping University Hospital for their efforts, and Caroline Brommesson for useful help with the ellipsometry.

\section{References}

1 Elkon K, Casali P. Nature and functions of autoantibodies. Nat Clin Pract Rheumato 2008; 4: 491-498.

2 Munoz LE, Lauber K, Schiller M, Manfredi AA, Herrmann M. The role of defective clearance of apoptotic cells in systemic autoimmunity. Nat Rev Rheumatol 2010; 6: 280-289.

3 Skogh T, Stendahl O. Complement-mediated delay in immune complex clearance from the blood owing to reduced deposition outside the reticuloendothelial system. Immunology 1983; 49 : 53-59.

4 Johansson AG, Lovdal T, Magnusson KE, Berg T, Skogh T. Liver cell uptake and degradation of soluble immunoglobulin $\mathrm{G}$ immune complexes in vivo and in vitro in rats. Hepatology (Baltimore, MD) 1996; 24: 169-175

5 Sjowall C, Olin AI, Skogh T, et al. C-reactive protein, immunoglobulin $\mathrm{G}$ and complement co-localize in renal immune deposits of proliferative lupus nephritis. Autoimmunity 2013; 46: 205-214.

6 Munoz LE, Janko C, Grossmayer GE, et al. Remnants of secondarily necrotic cells fuel inflammation in systemic lupus erythematosus. Arthritis Rheum 2009; 60: 1733-1742.

7 Munoz LE, Janko C, Chaurio RA, Schett G, Gaipl US, Herrmann M. IgG opsonized nuclear remnants from dead cells cause systemic inflammation in SLE. Autoimmunity 2010; 43: 232-235.

8 Jefferis R, Lund J, Pound JD. IgG-Fc-mediated effector functions: molecular definition of interaction sites for effector ligands and the role of glycosylation. Immunol Rev 1998; 163: 59-76.

9 Wuhrer M, Stam JC, van de Geijn FE, et al. Glycosylation profiling of immunoglobulin $\mathrm{G}(\mathrm{IgG})$ subclasses from human serum. Proteomics 2007; 7: 4070-4081.

10 Wang J, Balog CI, Stavenhagen K, et al. Fc-glycosylation of IgG1 is modulated by B-cell stimuli. MCP 2011; 10: M110.004655.

11 Huhn C, Selman MH, Ruhaak LR, Deelder AM, Wuhrer M. IgG glycosylation analysis. Proteomics 2009; 9: 882-913.

12 Arnold JN, Wormald MR, Sim RB, Rudd PM, Dwek RA. The impact of glycosylation on the biological function and structure of human immunoglobulins. Annu Rev Immunol 2007; 25: 21-50.

13 Qian J, Liu T, Yang L, Daus A, Crowley R, Zhou Q. Structural characterization of N-linked oligosaccharides on monoclonal antibody cetuximab by the combination of orthogonal matrix-assisted laser desorption/ionization hybrid quadrupole-quadrupole timeof-flight tandem mass spectrometry and sequential enzymatic digestion. Anal Biochem 2007; 364: 8-18.

14 Molinari M. N-glycan structure dictates extension of protein folding or onset of disposal. Nature Chem Biol 2007; 3: 313-320.

15 Aebi M, Bernasconi R, Clerc S, Molinari M. N-glycan structures: recognition and processing in the ER. Trends Biochem Sci 2010; 35: 74-82.

16 Nandakumar KS, Collin M, Olsen A, et al. Endoglycosidase treatment abrogates $\mathrm{IgG}$ arthritogenicity: importance of IgG glycosylation in arthritis. Eur J Immunol 2007; 37: 2973-2982.

17 Siberil S, de Romeuf C, Bihoreau N, et al. Selection of a human anti-RhD monoclonal antibody for therapeutic use: impact of $\operatorname{IgG}$ glycosylation on activating and inhibitory Fc gamma R functions. Clin Immunol 2006; 118: 170-179.

18 Peipp M, Lammerts van Bueren JJ, Schneider-Merck T, et al. Antibody fucosylation differentially impacts cytotoxicity mediated by NK and PMN effector cells. Blood 2008; 112: 2390-2399.

19 Anthony RM, Nimmerjahn F. The role of differential IgG glycosylation in the interaction of antibodies with FcgammaRs in vivo. Curr Opin Organ Transplant 2011; 16: 7-14.
20 Umana P, Jean-Mairet J, Moudry R, Amstutz H, Bailey JE. Engineered glycoforms of an antineuroblastoma IgG1 with optimized antibody-dependent cellular cytotoxic activity. Nature Biotechnol 1999; 17: 176-180.

21 Shields RL, Lai J, Keck R, et al. Lack of fucose on human IgG1 $\mathrm{N}$-linked oligosaccharide improves binding to human Fcgamma RIII and antibody-dependent cellular toxicity. J Biol Chem 2002; 277: 26733-26740.

22 Williams PJ, Arkwright PD, Rudd P, et al. Short communication: selective placental transport of maternal IgG to the fetus. Placenta 1995; 16: 749-756.

23 Kaneko Y, Nimmerjahn F, Ravetch JV. Anti-inflammatory activity of immunoglobulin $\mathrm{G}$ resulting from Fc sialylation. Science 2006; 313: 670-673.

24 Chen S, Lu C, Gu H, et al. Aleuria Aurantia Lectin (AAL)-reactive immunoglobulin $\mathrm{G}$ rapidly appears in sera of animals following antigen exposure. PloS One 2012; 7: e44422.

25 Tan EM, Cohen AS, Fries JF, et al. The 1982 revised criteria for the classification of systemic lupus erythematosus. Arthritis and rheumatism 1982; 25: 1271-1277.

26 Petri M, Orbai AM, Alarcon GS, et al. Derivation and validation of the Systemic Lupus International Collaborating Clinics classification criteria for systemic lupus erythematosus. Arthritis Rheum 2012; 64: 2677-2686

27 Gladman DD, Ibanez D, Urowitz MB. Systemic lupus erythematosus disease activity index 2000. J Rheumatol 2002; 29: 288-291.

28 Elwing $\mathrm{H}$. Protein absorption and ellipsometry in biomaterial research. Biomaterials 1998; 19: 397-406.

29 Sjowall C, Wettero J, Bengtsson T, et al. Solid-phase classical complement activation by $\mathrm{C}$-reactive protein (CRP) is inhibited by fluid-phase CRP-C1q interaction. Biochem Biophys Res Commun 2007; 352: 251-258.

30 Skoglund C, Wettero J, Skogh T, Sjowall C, Tengvall P, Bengtsson T. $\mathrm{C}$-reactive protein and $\mathrm{Clq}$ regulate platelet adhesion and activation on adsorbed immunoglobulin $\mathrm{G}$ and albumin. Immunol Cell Biol 2008; 86: 466-474.

31 Lood C, Allhorn M, Lood R, et al. IgG glycan hydrolysis by endoglycosidase $\mathrm{S}$ diminishes the proinflammatory properties of immune complexes from patients with systemic lupus erythematosus: a possible new treatment? Arthritis Rheum 2012; 64: 2698-2706.

32 Forger F, Matthias T, Oppermann M, Becker H, Helmke K Clinical significance of anti-dsDNA antibody isotypes: $\mathrm{IgG} / \mathrm{IgM}$ ratio of anti-dsDNA antibodies as a prognostic marker for lupus nephritis. Lupus 2004; 13: 36-44.

33 Gaipl US, Munoz LE, Grossmayer G, et al. Clearance deficiency and systemic lupus erythematosus (SLE). J Autoimmun 2007; 28 : 114-121.

34 Baumann I, Kolowos W, Voll RE, et al. Impaired uptake of apoptotic cells into tingible body macrophages in germinal centers of patients with systemic lupus erythematosus. Arthritis Rheum 2002; 46: 191-201.

35 Munoz LE, Janko C, Schulze C, et al. Autoimmunity and chronic inflammation - two clearance-related steps in the etiopathogenesis of SLE. Autoimmun Rev 2010; 10: 38-42.

36 Albert H, Collin M, Dudziak D, Ravetch JV, Nimmerjahn F. In vivo enzymatic modulation of $\mathrm{IgG}$ glycosylation inhibits autoimmune disease in an IgG subclass-dependent manner. Proc Nat Acad Sci 2008; 105: 15005-15009.

37 Van Beneden K, Coppieters K, Laroy W, et al. Reversible changes in serum immunoglobulin galactosylation during the immune response and treatment of inflammatory autoimmune arthritis. Ann Rheum Dis 2009; 68: 1360-1365.

38 Gornik I, Maravic G, Dumic J, Flogel M, Lauc G. Fucosylation of $\mathrm{IgG}$ heavy chains is increased in rheumatoid arthritis. Clin Biochem 1999; 32: 605-608.

39 van de Geijn FE, Wuhrer M, Selman MH, et al. Immunoglobulin $\mathrm{G}$ galactosylation and sialylation are associated with pregnancyinduced improvement of rheumatoid arthritis and the postpartum flare: results from a large prospective cohort study. Arthritis Res Ther 2009; 11: R193.

40 Bilyy R, Stoika R. Search for novel cell surface markers of apoptotic cells. Autoimmunity 2007; 40: 249-253. 
41 Bilyy RO, Shkandina T, Tomin A, et al. Macrophages discriminate glycosylation patterns of apoptotic cell-derived microparticles. J Biol Chem 2012; 287: 496-503.

42 Blaess S, Kammerer RA, Hall H. Structural analysis of the sixth immunoglobulin-like domain of mouse neural cell adhesion molecule L1 and its interactions with alpha(v)beta3, alpha(IIb)beta3, and alpha5betal integrins. J Neurochem 1998; 71: $2615-2625$.

43 Gornik O, Lauc G. Glycosylation of serum proteins in inflammatory diseases. Dis Markers 2008; 25: 267-278.

44 Raju TS. Terminal sugars of Fc glycans influence antibody effector functions of IgGs. Curr Opin Immunol 2008; 20: 471-478.

45 Swaak AJ, van Rooyen A, Vogelaar C, Pillay M, Hack E. Complement (C3) metabolism in systemic lupus erythematosus in relation to the disease course. Rheumatol Int 1986; 6: 221-226.
46 Satoh M, Iida S, Shitara K. Non-fucosylated therapeutic antibodies as next-generation therapeutic antibodies. Expert Opin Biol Ther 2006; 6: 1161-1173.

47 Zhou Q, Shankara S, Roy A, et al. Development of a simple and rapid method for producing non-fucosylated oligomannose containing antibodies with increased effector function. Biotechnol Bioeng 2008; 99: 652-665.

48 Wright A, Sato Y, Okada T, Chang K, Endo T, Morrison S. In vivo trafficking and catabolism of IgG1 antibodies with $\mathrm{Fc}$ associated carbohydrates of differing structure. Glycobiology 2000; 10: 1347-1355.

49 Saevarsdottir S, Steinsson K, Ludviksson BR, Grondal G, Valdimarsson H. Mannan-binding lectin may facilitate the clearance of circulating immune complexes-implications from a study on C2-deficient individuals. Clin Exp Immunol 2007; 148: 248-253. 